\title{
COMPLEX ORGANIC MATERIALS IN THE HR 4796A DISK?
}

\author{
M. Köhler, ${ }^{1}$ I. Mann, ${ }^{2}$ And Aigen Li ${ }^{1}$ \\ Received 2008 June 24; accepted 2008 September 2; published 2008 September 22
}

\begin{abstract}
The red spectral shape of the visible to near-infrared reflectance spectrum of the sharply edged ringlike disk around the young main-sequence star HR 4796A was recently interpreted as the presence of tholin-like complex organic materials which are seen in the atmosphere and surface of Titan and the surfaces of icy bodies in the solar system. However, we show in this Letter that porous grains composed of common cosmic dust species (amorphous silicate, amorphous carbon, and water ice) also closely reproduce the observed reflectance spectrum, suggesting that the presence of complex organic materials in the HR 4796A disk is still not definitive.
\end{abstract}

Subject headings: circumstellar matter — dust, extinction — infrared: stars -

planetary systems: protoplanetary disks — stars: individual (HR 4796A)

\section{INTRODUCTION}

HR $4796 \mathrm{~A}$ is a nearby (distance to the Earth $d \approx 67 \pm 3$ pc) young main-sequence (MS) star (age $\approx 8 \pm 3 \mathrm{Myr}$; Stauffer et al. 1995) of spectral type A0 V (effective temperature $T_{\text {eff }} \approx$ $9500 \mathrm{~K}$ ) with a large infrared (IR) excess which has recently aroused considerable interest. Imaging observations describe dust scattering in the optical (Debes et al. 2008) and near-IR (Augereau et al. 1999; Schneider et al. 1999) and thermal emission at mid-IR (Jayawardhana et al. 1998; Koerner et al. 1998; Telesco et al. 2000; Wahhaj et al. 2005). They reveal a ringlike disk with maximum at $\sim 70 \mathrm{AU}$ distance from the central star and $\sim 17 \mathrm{AU}$ width that is sharply truncated at the inner and the outer edge. The structure of the HR 4796A disk has important implications for planetesimal evolution (Kenyon et al. 1999). Furthermore, possibly existing planets may generate disk asymmetries through gravitational confinement or perturbation (Wyatt et al. 1999) or form rings with sharp edges (Augereau et al. 1999; Klahr \& Lin 2001; Thébault \& Wu 2008).

Very recently, Debes et al. (2008) measured a visible to nearIR photometric reflectance spectrum of the dust ring around HR 4796A. To fit the observed spectrum (which is characterized by a steep red slope increasing from $\lambda \approx 0.5$ to $1.6 \mu \mathrm{m}$ followed by a flattening of the spectrum at $\lambda>1.6 \mu \mathrm{m}$ ), Debes et al. (2008) argued for the presence of tholin-like organic material in the disk around HR 4796A. Tholin, a complex organic material, was detected as a major constituent of the atmosphere and surface of Titan and the surfaces of icy bodies in the solar system. The detection of tholin in the HR 4796A disk, if confirmed, would imply that these potential basic building blocks of life may be common in extrasolar planetary systems as well (see van Dishoeck 2008 for an overview of organic matter in space). Its young age places HR 4796A at a transitional stage between massive gaseous protostellar disks around young pre-MS T Tauri and Herbig Ae/Be stars ( 1 Myr) and evolved and tenuous debris disks around MS "Vega type" stars ( 100 Myr; see Jura et al. 1993; Chen \& Kamp 2004). Detecting organic matter in this system will provide valuable information about the formation and evolution of planetary systems.

The observed thermal emission of the disk, however, was previously reproduced with a model population of porous

\footnotetext{
${ }^{1}$ Department of Physics and Astronomy, University of Missouri, Columbia, MO 65211; koehlerme@missouri.edu, LiA@missouri.edu.

${ }^{2}$ School of Science and Engineering, Kindai University, Osaka 577-8502, Japan; mann@kindai.ac.jp.
}

grains consisting of the common cosmic dust species amorphous silicate, amorphous carbon, and water ice (Li \& Lunine 2003a). In this Letter we question the existence of tholin as a major dust component in the HR 4796A disk and study alternative dust models to reproduce the observed reflectance spectrum.

\section{MODEL}

Dust in the disk around HR 4796A scatters starlight at visible to near-IR wavelengths and emits thermally in the IR. The total flux of the disk $F_{\text {disk }}(\lambda)$ is the sum of the scattered light $F_{\text {sca }}(\lambda)$ and the dust thermal emission $F_{\text {therm }}(\lambda)$. At $\lambda<2.2$ $\mu \mathrm{m}$ the dominant contribution to $F_{\text {disk }}(\lambda)$ comes from the starlight scattered by dust $F_{\text {sca }}(\lambda)$ which is calculated from

$$
\begin{aligned}
& F_{\text {sca }}(\lambda)= \\
& \frac{F_{\lambda}^{\star}}{4 \pi d^{2}} \int_{r_{\text {in }}}^{r_{\text {out }}}\left(\frac{R_{\star}}{2 r}\right)^{2} \sigma(r) 2 \pi r d r \int_{a_{\min }}^{a_{\text {max }}} C_{\text {sca }}(\lambda, a) \Phi(\lambda, a) n(a) d a,
\end{aligned}
$$

where $d \approx 67 \mathrm{pc}$ is the distance from the star to the Earth; $F_{\lambda}^{*}$ is the stellar atmospheric flux approximated by the Kurucz model for A0 V stars with $T_{\text {eff }}=9500 \mathrm{~K}, \lg g=4.5$, and a solar metallicity (Kurucz 1979); $R_{\star}=1.7 R_{\odot}$ is the stellar radius; $r_{\text {in }}$ and $r_{\text {out }}$ are respectively the inner and outer boundaries of the disk; $\sigma(r)$ is the dust surface density distribution; $C_{\text {sca }}(\lambda, a)$ is the scattering cross section of spherical dust of radius $a$ at wavelength $\lambda ; \Phi(\lambda, a)$ is the phase function approximated by the Henyey-Greenstein function at a scattering angle of $\theta=90^{\circ}$ (see Debes et al. 2008) with the asymmetry parameter $g$ calculated from Mie theory for dust of size $a ;^{3}$ $n(a)$ is the dust size distribution which is taken to be a power law $n(a) \propto a^{-\alpha}$ with a lower cutoff $a_{\min }$, an upper cutoff

\footnotetext{
${ }^{3}$ The asymmetry parameters $g$ of the best-fit models averaged over the size distributions show a gradual decrease from $g \approx 0.96$ at $\lambda=0.55 \mu \mathrm{m}$ to $g \approx$ 0.85 at $\lambda=2.5 \mu \mathrm{m}$, indicating that the dust is highly forward-throwing. We should note that one should not compare the $g$ values calculated here with that of Debes et al. (2008), derived from the observed surface brightness of the disk together with the Henyey-Greenstein phase function. The former specify the degree of scattering in the forward direction $\left(\theta=0^{\circ}\right)$ of the dust (see Li 2008), while the latter approximate the mean scattering properties of the dust averaged over the entire disk.
} 


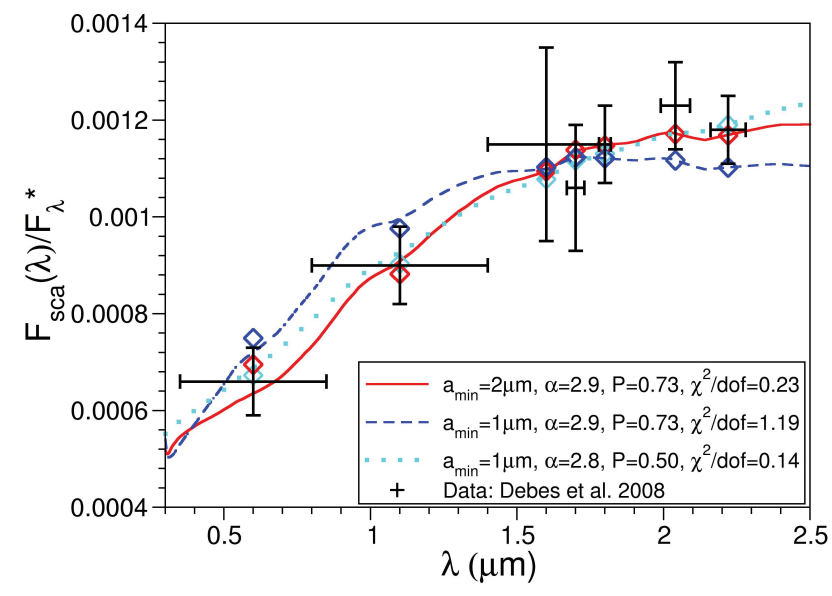

FIG. 1.-Comparison of the model scattered light spectra with the observed visible to near-IR reflectance spectrum of the HR 4796A disk of Debes et al. (2008; plus signs). Diamonds show the model spectra convolved with the HST STIS and NICMOS filters. The model with $P=0.73, a_{\min }=2 \mu \mathrm{m}$, and $\alpha=2.9$ (red solid line) is preferred since it fits both the observed reflectance spectrum and the observed IR emission (see Fig. 3). While the model with $P=0.50, a_{\min }=1 \mu \mathrm{m}$, and $\alpha=2.8$ (cyan dotted line) fits the reflectance spectrum better than any other models, it does not fit the observed IR emission (see Fig. 3). In contrast, the model with $P=0.73, a_{\min }=1 \mu \mathrm{m}$, and $\alpha=$ 2.9 (blue dashed line) provides an excellent fit to the observed IR emission; its fit to the observed reflectance spectrum is not as good as the other two models shown in this figure.

$a_{\max }$, and a power-law index $\alpha$. We take $a_{\max }=1000 \mu \mathrm{m}$ and treat $a_{\min }$ and $\alpha$ as free parameters.

Following Kenyon et al. (1999), Klahr \& Lin (2001), and Li \& Lunine (2003a), we approximate the dust surface density distribution by a Gaussian-type function $\sigma(r)=\sigma_{p} \exp \{-4 \times$ $\left.\ln 2\left[\left(r-r_{p}\right) / \Delta\right]^{2}\right\}$ where $r_{p}$ is the radial position where $\sigma(r)$ peaks, $\Delta$ is the full width at half-maximum (FWHM) of the distribution, and $\sigma_{p}$ is the midplane surface density at $r=r_{p}$. We take $r_{p}=70 \mathrm{AU}$ and $\Delta=15 \mathrm{AU}$ (see $\S 2.1$ of Li \& Lunine 2003a). We take $r_{\text {in }}=40 \mathrm{AU}$ and $r_{\text {out }}=100 \mathrm{AU}$ since there is little dust even at $r<55 \mathrm{AU}$ or $r>85 \mathrm{AU}$ (see Fig. 5 of Li $\&$ Lunine 2003a).

We consider porous grains composed of common dust species (amorphous silicate, amorphous carbon, ${ }^{4}$ and water ice; see Li \& Lunine 2003a). The optical properties of porous dust are determined with Mie theory in combination with the Bruggeman effective medium theory (Bohren \& Huffman 1983; see eqs. [7]-[9] of Li \& Lunine [2003b] for a detailed description). We take the mass ratio of amorphous carbon to amorphous silicate to be $m_{\text {carb }} / m_{\text {sil }}=0.7$ and the mass ratio of water ice to amorphous carbon and amorphous silicate to be $m_{\text {ice }} / m_{\text {carb }}+m_{\text {sil }}=0.8$, as inferred from the cosmic abundance constraints (see Appendix A of Li \& Lunine 2003a). Porous dust models consisting of fluffy aggregates of amorphous silicate, amorphous carbon, and water ice with such mixing ratios have been shown successful in reproducing the IR to submillimeter dust emission spectral energy distribution of the HR 4796A disk (Li \& Lunine 2003a; Sheret et al. 2004).

For amorphous silicate dust, we assume amorphous $(\mathrm{Mg}$, $\mathrm{Fe}) \mathrm{SiO}_{4}$, an amorphous material with olivine-normative composition for which we take optical constants from Jäger et al.

\footnotetext{
${ }^{4}$ The other carbon dust species widely considered in astrophysical modeling are graphite, hydrogenated amorphous carbon, quenched carbonaceous composite, and organic refractory. Their optical properties are not qualitatively different from that of amorphous carbon.
}

(1994). The optical constants of amorphous carbon and water ice are taken from Rouleau \& Martin (1991; "AC" type) and Warren (1984), respectively. Crystalline silicates are not included in our model calculations since observations at $\sim 8-13$ $\mu \mathrm{m}$ with the mid-IR Keck LWS show no crystalline silicate emission features (Kessler-Silacci et al. 2005). Although the optical properties of crystalline silicates differ from that of amorphous silicates, ${ }^{5}$ the resulting reflectivity is similar. Moreover, the amount of crystalline silicates, if observed in disks, is significantly smaller than that of the amorphous silicates.

A major characteristic of porous dust is its porosity $P$ (i.e., the fractional volume of vacuum in a porous grain). Li \& Lunine (2003a) have shown that the IR emission of the HR 4796A disk is best fit by dust with $P=0.90$. We should note that the best-fit porosity of $P=0.90$ refers to the porous aggregates of amorphous silicate and amorphous carbon; the porosity is reduced to $P \approx 0.73$ when ice fills in some of the vacuum under the assumption of a complete condensation of all condensable volatile elements as ice (see Appendix B of Li \& Lunine 2003a). It is expected that the dust in the HR 4796A disk will be coated by ice since at $\sim 70 \mathrm{AU}$ from the star the dust will be cooler than $\sim 110-120 \mathrm{~K}$ and ice condensation will occur (see Li \& Lunine 2003a). Assuming a complete ice condensation, the original porosity $P$ for the porous mixture of silicate and carbon will be reduced to $\max \{0,[1-$ $2.68(1-P)]\}$. Unless stated otherwise, in the following the porosity $P$ refers to the fractional volume of vacuum in icecoated porous dust. We will consider a range of porosities: $P=0,0.2,0.5,0.73,0.9$ (which correspond to $P \approx 0.63,0.70$, $0.81,0.90,0.96$ if ice is removed).

\section{RESULTS}

We compare $F_{\text {sca }}(\lambda) / F_{\lambda}^{\star}$ with the visible to near-IR reflectance spectrum of the HR 4796A disk compiled by Debes et al. (2008) either from archival images (HST STIS 50CCD at $\lambda=0.585$ $\mu \mathrm{m}, H S T$ NICMOS F110W at $\lambda=1.1 \mu \mathrm{m}$, F160W at $\lambda=$ $1.6 \mu \mathrm{m}$ ) or from their newly obtained $H S T$ NICMOS images (F171M, F180M, F204M, and F222M at $\lambda=1.71,1.80,2.04$, $2.22 \mu \mathrm{m}$, respectively).

By varying $a_{\min }$ and $\alpha$, we try to fit the observational data of Debes et al. (2008) with the porous dust model consisting of common dust species for which the mass mixing ratios are taken from the cosmic abundance considerations (see $\S 2$ ). We first take the porosity to be $P=0.73$ (which corresponds to $P=0.90$ for the porous dust without ice coating; the model with $P=0.90$ gives the best fit to the observed IR emission, as shown in Li \& Lunine 2003a). As illustrated in Figure 1, the model with $P=0.73, a_{\min } \approx 2 \mu \mathrm{m}$, and $\alpha \approx 2.9$ closely reproduces the reflectance spectrum of HR 4796A. In Figure 2 we plot the deviations of the model predictions from the HST photometry at each of the seven bands. The deviations are smaller than $\sim 7.5 \%$ for all wave bands. It is even more encouraging that the same model also closely fits the IR emission of this disk (see Fig. 3), except its slight deficiency at $\sim 10 \mu \mathrm{m}$ (but still well within the observational uncertainties). The model requires a total dust mass of $\sim 4.27 \times 10^{27} \mathrm{~g}$ and results

\footnotetext{
${ }^{5}$ While crystalline silicates in astrophysical regions are often found to be Mg-rich and Fe-poor (e.g., see Molster \& Kemper 2005), astronomical amorphous silicates appear to have a similar fraction of $\mathrm{Mg}$ and $\mathrm{Fe}$ as implied by the strong UV/visible absorptivity required to model the circumstellar emission (Jones \& Merrill 1976; Rogers et al. 1983) and interstellar extinction (Draine \& Lee 1984). This suggests that amorphous silicates could be less effective in scattering the visible to near-IR starlight.
} 


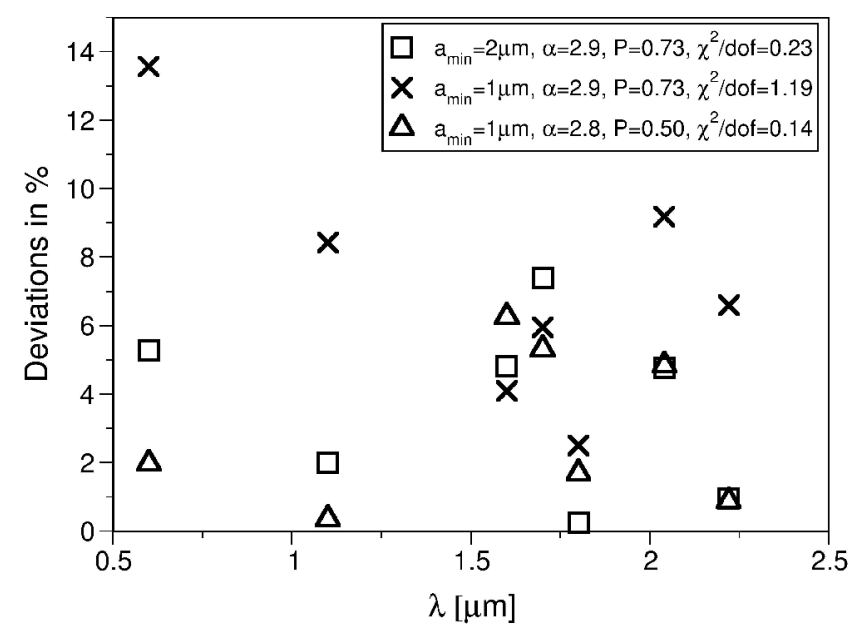

FIG. 2.-Deviations $\left|\left(F_{\text {sca }} / F_{\lambda}^{\star}\right)_{\text {mod }} /\left(F_{\text {sca }} / F_{\lambda}^{\star}\right)_{\text {obs }}-1\right|$ of the model results (integrated over the instrument filters) from the HST STIS and NICMOS photometry at each of the seven bands. The deviations are within $\sim 7.5 \%$ at all wave bands for the preferred model (with $P=0.73, a_{\min }=2 \mu \mathrm{m}$, and $\alpha=$ 2.9) which closely fits both the reflectance spectrum (see Fig. 1) and the IR emission of the HR 4796A disk (see Fig. 3).

in a vertical visible optical depth of $\tau_{V} \approx 0.048$ at $r=r_{p}$ and a midplane radial optical depth of $\tau_{V} \approx 0.56$. This justifies the optical thin approximation employed in this work.

In contrast, the model with $P=0.73, a_{\min } \approx 1 \mu \mathrm{m}$, and $\alpha \approx 2.9$ provides the best fit to the observed IR emission (see Li \& Lunine 2003a and Fig. 3). However, this model does not closely fit the observed reflectance spectrum (see Figs. 1 and 2 ), although the model reflectance spectrum does exhibit a general trend (i.e., a steep red slope at $\sim 0.5-1.6 \mu \mathrm{m}$ and subsequent flattening off; see Fig. 1) similar to that observed in the HR 4796A disk.

The best fit to the observed reflectance spectrum is given by the model with $P=0.50, a_{\min } \approx 1 \mu \mathrm{m}$, and $\alpha \approx 2.8$ (see Fig. 1). The deviations from the reflectance data are smaller than $\sim 6.2 \%$ for all wave bands (see Fig. 2). However, the model does not fit the observed IR emission (see Fig. 3).

Generally speaking, highly porous dust (with $P>0.85$ ) does not fit the observed reflectance spectrum well, while more compact dust (with $P<0.60$ ) is too cold to reproduce the observed IR emission. In Table 1 we list the parameters used in the different models: the minimal grain radius $a_{\min }$, the exponent of the size distribution $\alpha$, the porosity $P$, and $\chi^{2} /$ dof, where dof $\equiv$ $N_{\text {data }}-N_{\text {para }}$ is the "degree of freedom" $\left(N_{\text {data }}=7\right.$ is the number of data points at $\lambda=0.585,1.1,1.6,1.71,1.80,2.04,2.22 \mu \mathrm{m}$; $N_{\text {para }}=3$ is the number of free parameters: $a_{\min }, \alpha$, and $P$ ).

\section{DISCUSSION}

Debes et al. (2008) calculated the reflectance spectra of "astronomical silicates," water ice, hematite $\mathrm{Fe}_{2} \mathrm{O}_{3}$ (which is found on the surface of Mars and responsible for its redness), and UV laser ablated olivine (which has been used to explain the spectral reddening of silicate-rich asteroids due to space weathering; Brunetto et al. 2007). But the scattering spectra of these minerals and ice are too neutral at $\lambda \sim 0.5-1.6 \mu \mathrm{m}$ to match the steep red spectral slope of the reflectance spectrum of the HR 4796A disk. Debes et al. (2008) therefore resorted to organic materials. They found that tholin or its mixture with other dust species (e.g., water ice or olivine) are able to reproduce the observed red spectral slope. This led them to suggest that "the presence of organic material

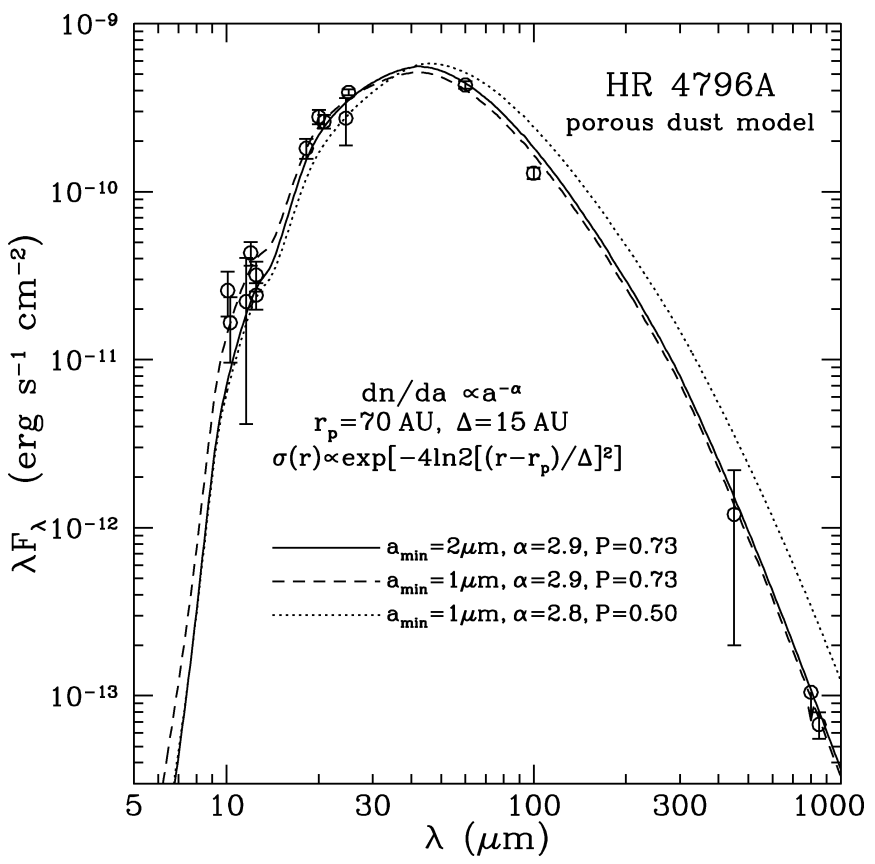

FIG. 3.-Comparison of the observed IR emission of the HR 4796A dust disk to the model spectra calculated from the porous dust models consisting of amorphous silicate, amorphous carbon, and ice.

is the most plausible explanation for the observations," with a cautionary note that "... longer wavelength scattered light observations will further constrain the (tholin-based) grain models, particularly around 3.8-4 $\mu \mathrm{m}$, where a large absorption feature is seen for different grain sizes of tholins. This would help to directly confirm whether Titan tholins are an adequate proxy for the material in orbit around HR 4796A."

However, as shown in Figure 1, simple porous dust models consisting of dust species (amorphous silicate, amorphous carbon, water ice) which are commonly considered to dominate in the interstellar medium (ISM), envelopes around evolved stars, and dust disks around young stars closely reproduce the observed reflectance spectrum of the HR 4796A disk. Our model provides at least a viable alternative to the tholin-based models of Debes et al. (2008). While the tholin organic dust model predicts a strong feature around 3.8-4 $\mu \mathrm{m}$ characteristic of tholin (Debes et al. 2008), the porous dust model presented here predicts a strong band at $\sim 3.1 \mu \mathrm{m}$, attributed to the $\mathrm{O}-\mathrm{H}$ stretching mode of water ice.

The tholin organics of which the optical constants were adopted by Debes et al. (2008) were made from DC discharge of $90 \% \mathrm{~N}_{2}$

TABLE 1

Model Parameters

\begin{tabular}{lcccc}
\hline \hline $\begin{array}{c}\text { Dust } \\
\text { Model }\end{array}$ & $\begin{array}{c}a_{\min } \\
(\mu \mathrm{m})\end{array}$ & $\alpha$ & $\begin{array}{c}\text { Porosity } \\
(\%)\end{array}$ & $\chi^{2} /$ dof \\
\hline $1 \ldots \ldots \ldots \ldots \ldots$ & 1 & 2.8 & 0 & 0.47 \\
$2 \ldots \ldots \ldots \ldots \ldots$ & 1 & 2.8 & 20 & 0.33 \\
$3 \ldots \ldots \ldots \ldots \ldots$ & 1 & 2.8 & 50 & 0.14 \\
$4 \ldots \ldots \ldots \ldots$. & 1 & 2.9 & 73 & 1.19 \\
$\mathbf{5} \ldots \ldots \ldots \ldots$. & $\mathbf{2}$ & $\mathbf{2 . 9}$ & $\mathbf{7 3}$ & $\mathbf{0 . 2 3}$ \\
$6 \ldots \ldots \ldots \ldots$ & 4 & 3.2 & 90 & 1.74 \\
\hline
\end{tabular}

NoTES. - Parameters for porous dust models consisting of common dust species (amorphous silicate, amorphous carbon, water ice). Model 5 is preferred since it closely reproduces both the observed reflectance spectrum (see Fig. 1) and the observed IR emission (see Fig. 3). Although model 3 fits the observed reflectance spectrum better than any other models (see Fig. 1), it does not fit the observed IR emission (see Fig. 3). 
and $10 \% \mathrm{CH}_{4}$ gas mixture (Khare et al. 1984). They are extremely $\mathrm{N}$-rich and optically very different from amorphous carbon. The dust in the HR 4796A disk should be continuously replenished. This is indicated by the low size cutoff $a_{\text {min }}$ (of a few micrometers) of the dust required to reproduce the reflectance spectrum (see $\S 2$ and Table 1) combined with considerations of dust lifetimes based on the radiation pressure and Poynting-Robertson effects (see Fig. 9 of Li \& Lunine 2003a). The replenishing source would likely arise from collisional cascades of larger bodies like planetesimals, asteroid-like and comet-like bodies. We suggest, with interstellar dust as the building blocks of the parent bodies, it is more reasonable to assume that the dust in the HR 4796A disk is composed of amorphous silicate, amorphous carbon, and water ice. ${ }^{6}$

The model which best fits both the observed reflectance spectrum and the IR emission requires highly porous dust (with $P=0.73$ which corresponds to $P=0.90$ if ice is sublimated; see $\S 2$ ). While it is natural to recognize that cold conglomeration of dust grains in molecular clouds can lead to highly porous dust structures, ${ }^{7}$ at a first glance, it is harder to accept that comparatively more violent collisions between larger bodies would result in such a morphology in the resulting debris.

To address this concern, we take the interplanetary dust particles (IDPs) as an analog for the dust in debris disks. The anhydrous chondritic IDPs collected in the stratosphere possibly of cometary origin show a highly porous structure (Brownlee 1987). Love et al. (1994) have measured the densities of $\sim 150$ unmelted chondritic IDPs with diameters of $\sim 5-15 \mu \mathrm{m}$, using grain masses determined from an absolute X-ray analysis technique with a transmission electron microscope and grain volumes determined from scanning elec-

\footnotetext{
${ }^{6}$ The tholin model may still be viable if the dust in the HR 4796A disk originates from the surface layers of planetesimals, possibly through excavating collisions (J. H. Debes 2008, private communication). In this scenario, the entire planetesimal need not be composed of tholins; they might reside only on the surface where they are created and then be released. Due to significant processing and possible alteration through planetesimal formation, the surface compositions of planetesimals in the HR 4796A disk may not resemble the ISM composition. Based on what we know from our own solar system, methane ice and water ice may reside on the surfaces of large planetesimals. These ices are exposed to the stellar UV flux and may ultimately produce tholin-like organic residues.

${ }^{7}$ A porosity in the range of $0.80 \leqq P \leqq 0.97$ is expected for dust aggregates formed through coagulation as shown both theoretically (Cameron \& Schneck 1965; Wada et al. 2008) and experimentally (Blum et al. 2006).
}

tron microscope imaging. They found that these particles have an average density of $\sim 2.0 \mathrm{~g} \mathrm{~cm}^{-3}$, corresponding to a moderate porosity of $\sim 0.4$. More recently, Joswiak et al. (2007) identified 12 porous cometary IDPs (based on their atmospheric entry velocities) with an average density of $\sim 1.0 \mathrm{~g} \mathrm{~cm}^{-3}$, corresponding to $P \approx$ 0.7 . Much higher porosities $(>0.9)$ have been reported for some very fluffy IDPs (e.g., MacKinnon et al. 1987; Rietmeijer 1993), despite that highly porous IDPs are probably too fragile to survive atmospheric entry heating. Low densities are also derived for different groups of meteoroids ${ }^{8}$ ascribed to comets: the densities are $\sim 1.0,0.75$, and $0.27 \mathrm{~g} \mathrm{~cm}^{-3}$, respectively (Ceplecha et al. 1998), corresponding to a porosity of $\sim 0.7,0.8$, and $>0.9$.

It is therefore reasonable to assume that the dust in the HR 4796A disk has a high porosity (at least for those originated from cometary bodies). We are not sure about the relative contributions to the HR 4796A disk from asteroid collisions and cometary activity. Note that this is a long-standing problem even for our own solar system (e.g., see Lisse 2002).

To conclude, we argue that the presence of tholin-like complex organic materials in the HR 4796A disk is still not conclusive since the observed red spectral shape of the disk can be closely reproduced by models of porous dust comprised of common cosmic dust species. A more thorough study of the scattered light over a range of scattering angles would further constrain the optical properties of the dust.

We thank J. H. Debes and the anonymous referee for their very helpful comments. M. K. and A. L. are supported in part by NASA/HST Theory Programs and NSF grant AST 0707866. A. L. is supported by the NSFC Outstanding Overseas Young Scholarship.

\footnotetext{
${ }^{8}$ By definition, meteoroids are small bodies in the mass range of $\sim 10^{-4}$ to $10^{8} \mathrm{~g}$, which orbit the Sun in interplanetary space. The atmospheric trajectories of meteors, i.e., the brightness generated by meteoroids passing through atmosphere, contain information about meteoroid orbits and densities. Meteoroids are accordingly classified into groups with different orbits, structure and composition. The densities given above are valid for between $\sim 50 \%$ and $73 \%$ of cometary meteor observations, depending on the observation method. The same studies show densities of $\sim 2 \mathrm{~g} \mathrm{~cm}^{-3}$ for the remaining cometary meteoroids, as well as for a significant part of the meteoroids ascribed to asteroids, which is also moderately porous (see Mann 2008).
}

\section{REFERENCES}

Augereau, J. C., et al. 1999, A\&A, 348, 557

Blum, J., et al. 2006, ApJ, 652, 1768

Bohren, C. F., \& Huffman, D. R. 1983, Absorption and Scattering of Light by Small Particles (New York: Wiley)

Brownlee, D. E. 1987, in Interstellar Processes, ed. D. J. Hollenbach \& H. A. Thronson (Dordrecht: Reidel), 513

Brunetto, R., Roush, T. L., Marra, A. C., \& Orofino, V. 2007, Icarus, 191, 381

Cameron, A. G. W., \& Schneck, P. B. 1965, Icarus, 4, 396

Ceplecha, Z., et al. 1998, Space Sci. Rev., 84, 327

Chen, C. H., \& Kamp, I. 2004, ApJ, 602, 985

Debes, J. H., Weinberger, A. J., \& Schneider, G. 2008, ApJ, 673, L191

Draine, B. T., \& Lee, H. M. 1984, ApJ, 285, 89

Jäger, C., et al. 1994, A\&A, 292, 641

Jayawardhana, R., et al. 1998, ApJ, 503, L79

Jones, T. W., \& Merrill, K. M. 1976, ApJ, 209, 509

Joswiak, D. J., Brownlee, D. E., Pepin, R. O., \& Schlutter, D. J. 2007, in Dust in Planetary Systems (ESA SP-643; Noordwijk: ESA), 141

Jura, M., Zuckerman, B., Becklin, E. E., \& Smith, R. C. 1993, ApJ, 418, L37

Kenyon, S. J., et al. 1999, ApJ, 524, L119

Kessler-Silacci, J. E., et al. 2005, ApJ, 622, 404

Khare, B. N., et al. 1984, Icarus, 60, 127

Klahr, H. H., \& Lin, D. N. C. 2001, ApJ, 554, 1095

Koerner, D. W., et al. 1998, ApJ, 503, L83

Kurucz, R. L. 1979, ApJS, 40, 1
Li, A. 2008, in Small Bodies in Planetary Sciences, ed. I. Mann, A. Nakamura, \& T. Mukai (Berlin: Springer), 167

Li, A., \& Lunine, J. I. 2003a, ApJ, 590, 368 2003b, ApJ, 594, 987

Lisse, C. M. 2002, Earth Moon Planets, 90, 497

Love, S. G., Joswiak, D. J., \& Brownlee, D. E. 1994, Icarus, 111, 227

MacKinnon, I. D. R., et al. 1987, Meteoritics, 22, 450

Mann, I. 2008, in Landolt Börnstein (New Series, Group VI, Vol. 2), in press

Molster, F., \& Kemper, C. 2005, Space Sci. Rev., 119, 3

Rietmeijer, F. J. M. 1993, Earth Planet. Sci. Lett., 117, 609

Rogers, C., Martin, P. G., \& Crabtree, D. R. 1983, ApJ, 272, 175

Rouleau, F., \& Martin, P. G. 1991, ApJ, 377, 526

Schneider, G., et al. 1999, ApJ, 513, L127

Sheret, I., Dent, W. R. F., \& Wyatt, M. C. 2004, MNRAS, 348, 1282

Stauffer, J. R., et al. 1995, ApJ, 454, 910

Telesco, C. M., et al. 2000, ApJ, 530, 329

Thébault, P., \& Wu, Y. 2008, A\&A, 481, 713

van Dishoeck, E. F. 2008, in Organic Matter in Space, ed. S. Kowk (Cambridge: Cambridge Univ. Press), in press

Wada, K., et al. 2008, ApJ, 677, 1296

Wahhaj, Z., et al. 2005, ApJ, 618, 385

Warren, S. G. 1984, Appl. Opt., 23, 1206

Wyatt, M. C., et al. 1999, ApJ, 527, 918 